\title{
Mammalian diaphanous-related formin 1 is required for motility and invadopodia formation in human U87 glioblastoma cells
}

\author{
ZHE LI, YAMING XU, CAN ZHANG, XIN LIU, LI JIANG and FUXUE CHEN \\ School of Life Sciences, Shanghai University, Shanghai 200444, P.R. China
}

Received August 2, 2013; Accepted November 13, 2013

DOI: $10.3892 /$ ijmm.2013.1577

\begin{abstract}
Characterized by invasive growth and infiltrative dissemination, glioma is poorly diagnosed and prognosed at present. The mammalian diaphanous-related formin 1 (mDRF1), which is involved in a number of actin-related biological processes, has been found to participate in the process of invasion and metastasis in human breast cancer cells and to show abnormal expression under pathological conditions. However, the role of mDRF1 in glioma is not clear. In this study, we carried out a comprehensive analysis of the effects of mDRF1 on human glioma. We used siRNA to knock down mDRF1 expression in highly invasive U87 malignant glioma (MG) cells and examined the changes in cell proliferation, apoptosis, invasion and migration. Atomic force microscopy was used to examine invadopodia formation. Immunohistochemical and immunocytochemical assays were used to analyze the cellular localization and the expression levels of mDRF1 in human glioma tissue and in the U87 MG cells. Following the transfection of U87 MG cells with siRNA-mDRF1, their in vitro proliferation was significantly decreased, apoptosis was markedly increased, and invasion and metastasis were significantly inhibited. The results from atomic force microscopy revealed that invadopodia were formed at leading the edge of the U87 MG cells. However, following the silencing of mDRF1 by siRNA, the edge of the cells became smooth and the invadopodia disappeared. For in vivo experiments, nude mice were transplanted with tumor cells and then treated with siRNA-mDRF1. The results revealed that treatment with siRNA-mDRF1 significantly inhibited tumor growth and led to a decrease in the weight of the transplanted tumor. In conclusion, our data demonstrate that mDRF1 is highly expressed in human glioma tissue. The knockdown of mDRF1 in U87 MG cells led to a sharp decline in their invasive and metastatic ability, which effectively reduced the spread of glioma cells into the surrounding areas. To our knowledge, this is the first report showing that mDRF1 is a promising target for the treatment of malignant gliomas.
\end{abstract}

Correspondence to: Professor Fuxue Chen, School of Life Sciences, Shanghai University, No. 333 Nanchen Road, Baoshan, Shanghai 200444, P.R. China

E-mail: chenfuxue@staff.shu.edu.cn

Key words: mammalian diaphanous-related formin 1, invasion, migration, invadopodia, glioblastoma

\section{Introduction}

As reported by the World Health Organization in 2007 (1), glioma is a common malignant tumor of the central nervous system, categorized in four grades based on histological features. Grade IV glioma, also termed glioblastoma multiforme (GBM), is the most common primary malignant brain tumor, resulting in a poorly demarcated interface between tumor and healthy brain tissues with no clear boundaries, which represents a serious obstacle in conventional treatment (2). Approximately 50\% of gliomas occupy more than one lobe of a hemisphere or are bilateral (3). Through finger-like tentacles, gliomas often spread to other parts of the brain and cause fatal damage to healthy brain tissues (4). The survival rate of patients with malignant glioma treated with conventional methods remains very low due to poor prognosis. Even when combining radiation therapy and concurrent chemotherapy with temozolomide, the median patient survival is limited to 14.6 months (5). Therefore, the identification of genes for effective targeting of malignant glioma is a priority in current research related to the treatment of this type of tumor.

As a protein with a key role in cytoskeletal rearrangements, the mammalian diaphanous-related formin 1 (mDRF1), also termed DIAPH1, is indispensable in microtubule formation and in tumor cell migration (6). The mDRF1 protein has two domains categorized as members of the formin family, formin homology 1 (FH1) and 2 (FH2) (7). The protein is involved in morphogenesis, cytokinesis, the formation of cell polarity, cell adhesion and migration, and shows anomalous expression during tumor differentiation $(8,9)$, as well as during the invasion and metastasis of MDA-MB-231 cells (10). However, very little is known about its roles in human glioma.

In highly invasive tumor cells, the actin-rich protrusions with extracellular matrix (ECM) proteolytic activity are termed invadopodia (11). Invadopodia have been found in a number of invasive malignant neoplasms, including head and neck squamous cell carcinoma, breast carcinoma and melanoma (12-14). Invadopodia that can accelerate the metastasis and invasion of malignant tumor cells in 3D Matrigel serve as the most important target in cancer treatment (15). Cortactin and phosphotyrosine are abundant in the invadopodia of malignant tumor cells (16). The potential association of invadopodia with mDRF1 remains unconfirmed.

In this study, we demonstrate that, as a vital component of glioma cells, mDRF1 plays a pivotal role during the invasion, 
metastasis, proliferation, apoptosis and invadopodia formation of human malignant glioma cells.

\section{Materials and methods}

Cell lines, human tissues and reagents. The U87 human malignant glioma (MG) cell line was purchased from the Chinese Academy of Sciences. Cells were cultured in Dulbecco's modified Eagle's medium (DMEM) supplemented with $10 \%$ fetal bovine serum (FBS) (both from Invitrogen Life Technologies, Carlsbad, $\mathrm{CA}, \mathrm{USA}$ ) at $37^{\circ} \mathrm{C}$ in a $5 \% \mathrm{CO}_{2}$ incubator.

Human glioma (grade IV) and healthy brain tissues were obtained from patients registered at the Renji Hospital of Shanghai Jiaotong University (Shanghai, China). Healthy brain tissues (mostly from the cortex) were collected from patients with physical brain injuries. Written informed consent was obtained from all patients. The use of human tissue was approved by the ethics committees of the hospital.

Nude mice were obtained from B\&K Universal Group Ltd. (Shanghai, China). All animals were treated in accordance with the principles and procedures of The Animal Care and Experimental Committee of the School of Medicine of Shanghai Jiao Tong University.

Rabbit monoclonal antibody for mDRF1 was purchased from Abcam Biosciences (Cambridge, MA, USA). Monoclonal mouse anti- $\beta$-actin, anti-cortactin and anti-phosphotyrosine antibodies were obtained from Cell Signaling Technology (Danvers, MA, USA).

Reagents and kits were purchased from: FuGENE ${ }^{\circledR} \mathrm{HD}$ Transfection reagent from Promega Corp. (Madison, WI, USA), Transwell kit for cell invasion assays from BD Biosciences (Franklin Lakes, NJ, USA), Annexin V-Cy3 apoptosis detection kit from BioVision Inc. (Milpitas, CA, USA), Cell Counting kit-8 (CCK-8) for proliferation assays from Dojindo Molecular Technologies Inc. (Kumamoto, Japan) and Radius ${ }^{\mathrm{TM}}$ 24-Well Cell Migration Assay kit from Cell Biolabs, Inc. (San Diego, CA, USA).

Constructs and transfection. The pGPU6-DIAPH1-1 plasmid (Shanghai GenePharma Co., Shanghai, China) was constructed and transfected into the U87 MG cells to knock down the expression of mDRF1. A random siRNA plasmid, pGPU6-DIAPH1-NC (Shanghai GenePharma Co.) was used as the negative control. Cells were seeded in 6-well plates at $1 \times 10^{6} /$ well and allowed to attach for $24 \mathrm{~h}$ prior to transfection. Subsequently, the pGPU6-DIAPH1-1 and pGPU6-DIAPH1-NC plasmids were transfected using the FuGENE ${ }^{\circledR}$ HD Transfection reagent according to the manufacturer's instructions. Following incubation for $48 \mathrm{~h}$ at $37^{\circ} \mathrm{C}$, cells were collected to study the expression of mDRF1 by western blot analysis.

Atomic force microscopy $(A F M)$. The atomic force microscope [also referred to as the scanning force microscope (SFM)] uses a microcantilever system to detect the atomic or molecular forces between the probe tip and the samples to generate images of samples. We used the 5500 AFM/SFM (Agilent Technologies, Santa Clara, USA) in its contacting mode in air at room temperature to obtain the topography AFM images. Rectangular nitrate silicon probes were used with nominal spring constant around $2.5 \mathrm{~N} / \mathrm{m}$ (Agilent Technologies) and microcantilever length of $120 \mu \mathrm{m}$. The microcantilever resonance frequency was approximately $30 \mathrm{kHz}$. The rms free amplitude of the cantilever was on the order of $15 \mathrm{~nm}$ and the relative set-point above $97 \%$ of the free amplitude. Images were recorded with a slow scan rate (below $1 \mathrm{~Hz}$ ).

Sample preparation for AFM imaging. Three groups of U87 MG cells were prepared for AFM imaging: untreated U87 MG cells, U87 MG cells transfected with the pGPU6-DIAPH1-1 plasmid and U87 MG cells transfected with the pGPU6-DIAPH1-NC plasmid. After $48 \mathrm{~h}$ of transfection, the medium was carefully removed without disturbing the cells. This was followed by the addition of $100 \%$ ethyl alcohol to the petri dish to fix the U87 MG cells for 15-20 min. The ethyl alcohol was then removed and the petri dish was allowed to to air dry.

Immunohistochemistry. The localization of mDRF1 in human healthy/glioma tissue was examined by immunohistochemical assays. Following the antibody-antigen interaction, the targeted protein was visualized via either chromogenic detection with diaminobenzidine (DAB) or fluorescent detection. We followed standard procedures, briefly: the primary antibody of mDRF1 was incubated with the tissue for $2 \mathrm{~h}$ at room temperature or overnight at $4^{\circ} \mathrm{C}$, to allow binding to the target antigen. A biotinylated secondary antibody, specific to mDRF1, was incubated with the tissue for $2 \mathrm{~h}$ at room temperature to allow binding to the primary antibody. A biotinylated enzyme (HRP) was pre-incubated with free avidin to form avidin-biotin-enzyme complexes. This solution was added to the tissue sections, and any remaining biotin-binding sites on the avidin bound to the biotinylated secondary antibody that had already bound to the tissue. The colored sites were observed under a confocal microscope.

Immunocytochemistry. After cleaning the poly-L-lysinecoated coverslips with ethanol, we plated the U87 MG cells at a density of $5 \times 10^{5}$ cells per coverslipped well and let cells grow overnight. The culture medium was then removed and the U87 MG cells were fixed with $4 \%$ formaldehyde for $20 \mathrm{~min}$ at room temperature. The fixative was aspirated and the cells were washed twice with PBS. The U87 MG cells were then incubated with PBST (PBS with $0.1 \%$ Triton X-100) for $30 \mathrm{~min}$. After washing with PBS three times, we blocked unspecific binding sites with $5 \%$ goat serum. The blocking buffer was then removed and the primary antibody was added followed by incubation at $4^{\circ} \mathrm{C}$ overnight. Subsequently, the primary antibody was removed and the cells were washed three times with PBST for $5 \mathrm{~min}$. The secondary antibody was incubated for $1 \mathrm{~h}$ in the dark and washed with PBST three times. Nuclear labeling was performed by incubating with the DNA-binding agent DAPI in dry form, followed by three 5-min washes with PBS. Fingernail polish was applied at the edges of the coverslip and the samples were examined under a confocal microscope.

Western blot analysis. Forty-eight hours after transfection, an equal number of U87 MG cells from each experimental group was used to extract total protein using RIPA lysis buffer. Electrophoresis was performed on a $10 \%$ SDS-PAGE gel and was followed by transfer onto a PVDF membrane. 
The membrane was then blocked by incubating with skim milk overnight at $4^{\circ} \mathrm{C}$. Following incubation with primary and secondly antibodies, the expression level of immunoreactive mDRF1 was detected using enhanced chemiluminescence (ECL) reagents (Cell Signaling Technology).

Proliferation assay. The assay was performed using the CCK-8 kit as follows: U87 MG cells were seeded in 96-well plates $\left(1 \times 10^{4}\right.$ cells/well) with six replicate wells for each group. Forty-eight hours after transfection, $10 \mu \mathrm{l}$ of WST-8 reagent \{[2-(2-methoxy-4-nitrophenyl)-3-(4-nitrophenyl)-5-(2,4-disulfophenyl)-2H-tetrazolium, monosodium salt]\} were added to each well and the cells were incubated in a $5 \% \mathrm{CO}_{2}$ incubator at $37^{\circ} \mathrm{C}$ for $1 \mathrm{~h}$. Cell proliferation, expressed as the cell survival rate, was analyzed in a microplate reader at $450 \mathrm{~nm}$ absorbance. Untransfected cells, cells transfected with pGPU6-DIAPH1NC and cells transfected with pGPU6-DIAPH1-1 were deemed as the untreated, the mDRF1 knockdown-negative and the mDRF1 knockdown or RNAi group, respectively.

Apoptosis assay. Following transfection for 48 or $72 \mathrm{~h}$, $4 \times 10^{5}$ U87 MG cells were collected by centrifugation. The Annexin $\mathrm{V}-\mathrm{Cy} 3$ reagent was then added and the cells were incubated at room temperature for $5 \mathrm{~min}$ in the dark. Apoptotic cells, the plasma membrane of which exhibited red fluorescence, were quantified under a fluorescent microscope in five randomly selected areas. The results are expressed as apoptotic rates. Untransfected cells served as the untreated negative control group, while the cells transfected with the pGPU6DIAPH1-1 plasmid were deemed as the RNAi group.

Wound healing assay. The assay was performed using the Radius $^{\mathrm{TM}}$ 24-Well Cell Migration Assay kit (Cell Biolabs, Inc.). Briefly, to monitor the migratory properties of the cells during wound healing $48 \mathrm{~h}$ after transfection, the U87 MG cells were seeded in 24-well plates until they reached $80-90 \%$ confluence. Each plate well contains a $0.68 \mathrm{~mm}$ non-toxic, biocompatible hydrogel spot in the center, where cells cannot attach. Once firm cell attachment was achieved, the hydrogel was rapidly removed to expose a cell-free region for cell migration This cell-free region in the center was the wound. Cells were photographed under a microscope at $0,2,5,8,11,18$ and $24 \mathrm{~h}$ after wound formation. The experiments were performed in triplicate. For the evaluation of the 'wound closure' area, we used Cell Profiler ${ }^{\mathrm{TM}}$ Cell Image Analysis software (available at: http://www.cellprofiler.org/).

Transwell assay. A cell invasion assay was performed using a 24-well Transwell chamber containing an $8-\mu$ pore size polyethylene glycol terephthalate (PET) membrane with a thin layer of Matrigel basement membrane matrix. Forty-eight hours after transfection, $1 \times 10^{4}$ U87 MG cells were trypsinized and transferred to the upper matrigel chamber in $500 \mu \mathrm{l}$ of serum-free DMEM medium. DMEM medium supplemented with $10 \%$ FBS was added to the lower chamber as the chemoattractant. Following incubation for $24 \mathrm{~h}$, cells remaining in the upper chamber were carefully removed with cotton swabs, while invading cells were fixed with dehydrated alcohol, stained with hematoxylin and eosin (H\&E), counted and photographed under a microscope by randomly selecting five x200 fields for each well. The experiment was repeated three independent times.

Tumor model in nude mice. Age-matched adult male nude mice, four weeks old, were housed in a temperature- and light-controlled environment with a 14/10-h light/dark cycle. The U87 MG cells were harvested in serum-free culture medium and the concentration of the cell suspension was adjusted to $5 \times 10^{7}$ viable cells per $\mathrm{ml}$. The cell suspension $(0.2 \mathrm{ml})$ was subcutaneously injected in the right lateral of the mice oxter. The tumor was formed within approximately 15 days after injection. The mice were randomly divided into three groups: i) the untreated group, where the mice were transplanted with untransfected cells; ii) the mDRF1 knockdown-negative group, where the mice were transplanted with cells transfected with the pGPU6-DIAPH1-NC plasmid; and iii) the mDRF1 knockdown/RNAi group, where the mice were transplanted with cells transfected with the pGPU6-DIAPH1-1 plasmid. Mice were observed daily and inspected every two days for the progression of tumor growth. After 25 days of observation, the mice were sacrificed and an autopsy was immediately performed. The tumor tissues were then fixed in $4 \%$ paraformaldehyde.

Statistical analysis. Data were processed using a one-way ANOVA and are presented as the means \pm standard error of the mean (SEM). The significance threshold for all tests was set to $\mathrm{P}<0.05$.

\section{Results}

Expression and distribution of mDRF1 in U87 MG cells. The human primary glioblastoma cell line, U87 MG, that shows epithelial morphology (grade IV) was used in our experiments. In order to analyze the distribution of mDRF1 in these cells, we performed an immunocytochemical staining assay. By using a biotinylated mDRF1-specific antibody, the expression and cellular distribution of mDRF1 was indicated by red fluorescence, which we found distributed in both the cell membrane and the cytoplasm (Fig. 1). By contrasting the red fluorescence associated with mDRF1 to the blue fluorescence emitted by DNA in the nucleus, mDRF1 was detected as expressed in the membrane as a cytoskeletal protein.

mDRF1 is overexpressed in human glioma tissue. In order to determine whether mDRF1 is overexpressed in human glioma tissue, we compared the expression levels of mDRF1 in human healthy tissue to malignant glioma brain tissue. Using the mDRF1-specific antibody, immunohistochemical and immunofluorescence assays were performed. Modena particles where mDRF1 was expressed in the malignant glioma tissue (Fig. 2B) markedly outnumbered those in the healthy brain tissue (Fig. 2A). This indicates that mDRF1 may be overexpressed in human glioma tissue. Fig. 3A shows the red fluorescent areas indicating the expression of $\mathrm{mDRF} 1$ following incubation with the mDRF1-specific antibody.

Successful knockdown of $m D R F 1$ protein expression in U87 MG cells. To confirm the transient transfection effects in the three groups of cells mentioned above, we measured the protein level of mDRF1 at $48 \mathrm{~h}$ after transfection. Western 

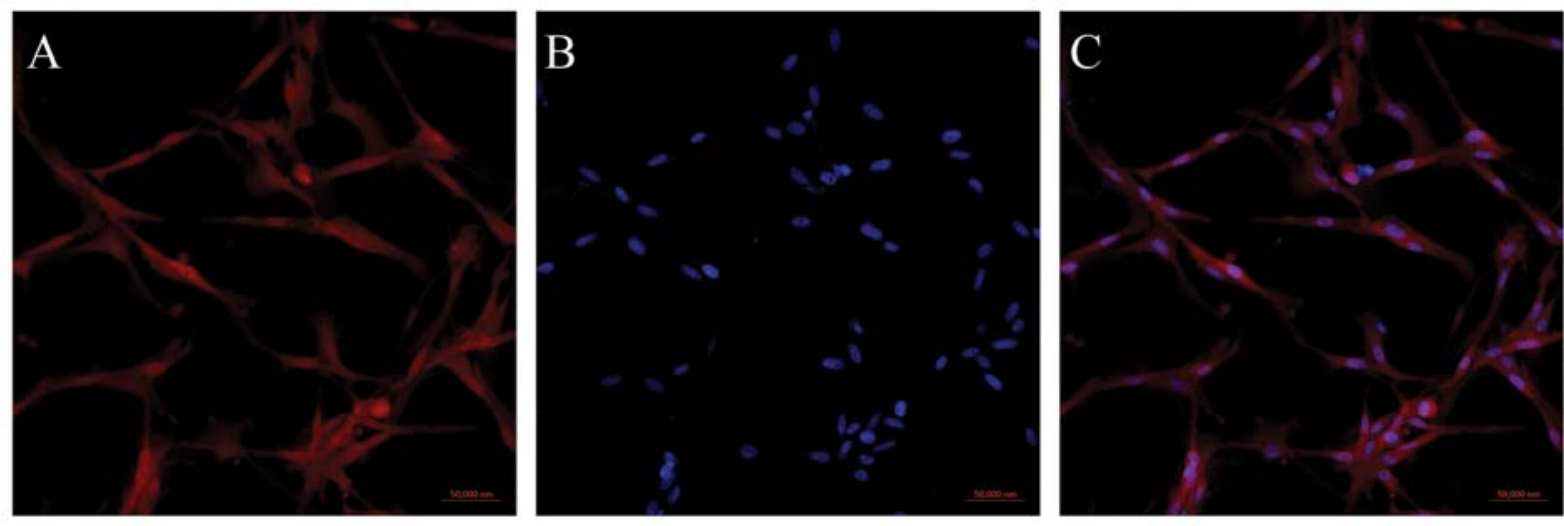

Figure 1. Mammalian diaphanous-related formin 1 (mDRF1) is expressed in the malignant glioma (MG) cell line, U87. (A) mDRF1 (red) was visualized with the anti-mDRF1 antibody. (B) The nucleus (blue) was visualized with DAPI. (C) Merge image of (A) and (B). Scale bar (A, B and C), 50,000 nm.
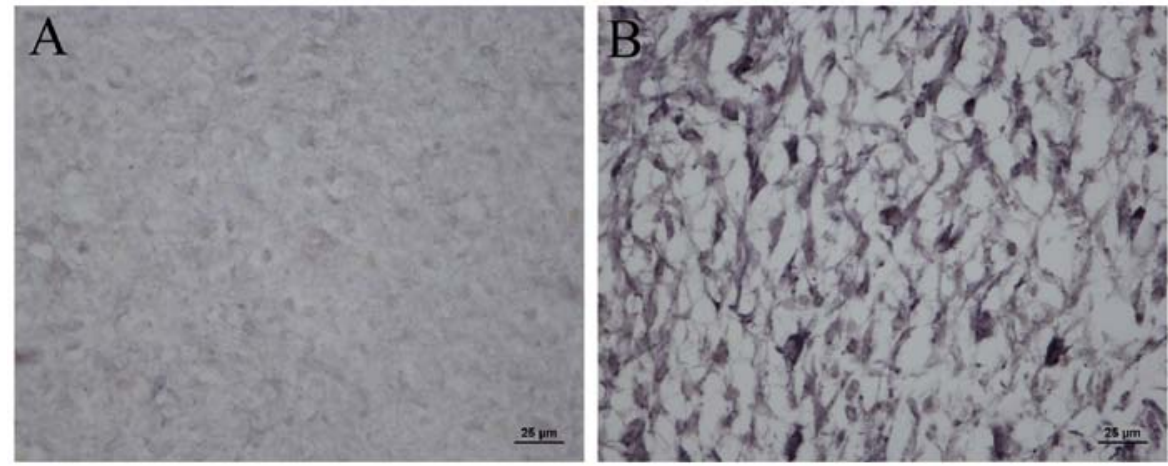

Figure 2. Representative examples of the immunohistochemical analysis using anti-mDRF1 antibody. (A) Normal and (B) malignant glioma brain tissue. Scale bar, $25 \mu \mathrm{m}$.
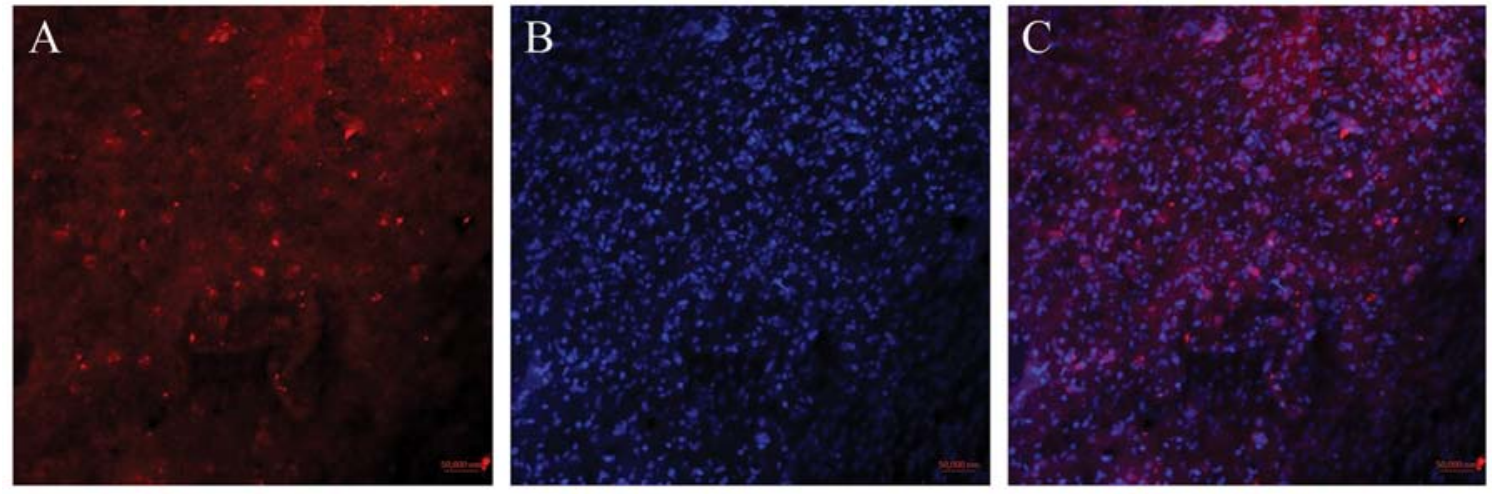

Figure 3. Mammalian diaphanous-related formin 1 (mDRF1) is expressed in human glioma tissue. (A) mDRF1 (red) was visualized with anti-mDRF1 antibody. (B) The nucleus (blue) was visualized with DAPI. (C) Merge image of (A) and (B). Scale bar (A, B and C), $50 \mu \mathrm{m}$.

blot analysis indicated that transient transfection efficiency was most predominant at $48 \mathrm{~h}$. The negative control groups (untreated and mDRF1 knockdown-negative) showed no difference in the mDRF1 protein level (Fig. 4). The level of the $\beta$-actin gene was used for normalization.

Knockdown of mDRF1 inhibits cell proliferation. After transfection for $48 \mathrm{~h}$ and incubation of the cells with WTS- 8 for $1 \mathrm{~h}$, the proliferation of the U87 MG cells was assessed. As illustrated in Fig. 5, the optical density (OD) value was 1.149 for the untransfected cells (untreated group), 1.112 for the cells transfected with the pGPU6-DIAPH1-NC plasmid (mDRF1 knockdown-negative group) and 0.747 for the U87 cells transfected with the pGPU6-DIAPH1-1 plasmid (RNAi group). The OD values of the untreated control group were significantly higher compared with the RNAi group, i.e., compared with the cells in which mDRF1 was knocked down. There was no obvious difference between the untreated and the mDRF1 knockdown-negative group. In conclusion, the knockdown of mDRF1 significantly reduced the proliferation ability of the U87 MG cells. These results indicate that mDRF1 is involved in U87 MG cell proliferation. 


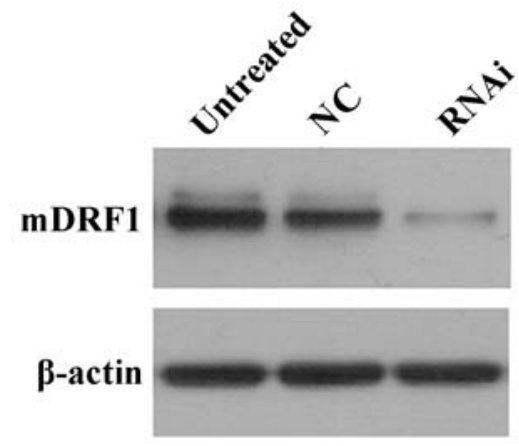

Figure 4. Protein level of mammalian diaphanous-related formin 1 (mDRF1) analyzed by western blot analysis at $48 \mathrm{~h}$ after transfection. The expression of mDRF1 markedly decreased in the RNAi (mDRF1 knockdown) group, transfected with pGPU6-DIAPH1-1, in contrast to the two negative control groups (untreated, untransfected cells; NC, mDRF1 knockdown-negative group). The level of the $\beta$-actin gene was used for normalization.

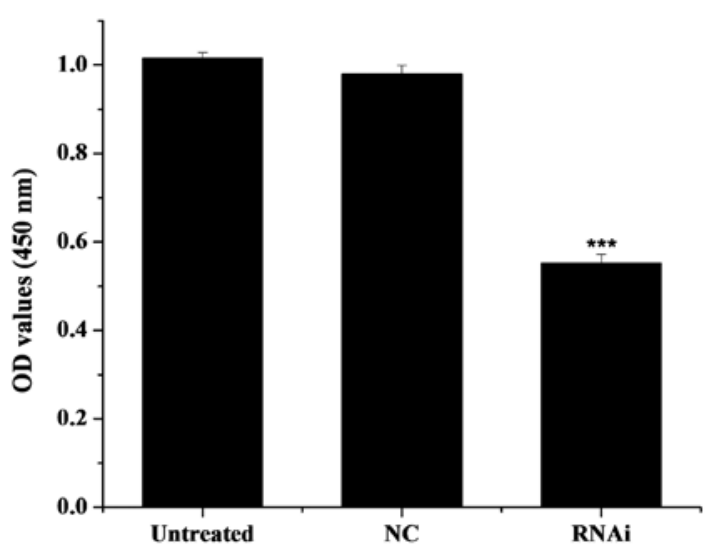

Figure 5. Proliferation assay of U87 malignant glioma (MG) cells using the cell counting kit-8 (CCK-8). Untreated, untransfected cells; NC, cells transfected with the pGPU6-DIAPH1-NC plasmid, as the mDRF1 knockdown-negative control group; RNAi, cells transfected with the pGPU6-DIAPH1-1 plasmid, as the mDRF1 knockdown group. Statistic comparisons were performed on $O D$ values measured at $450 \mathrm{~nm}$. Data are shown as the means $\pm \mathrm{SE},{ }^{* * *} \mathrm{P}<0.001$.

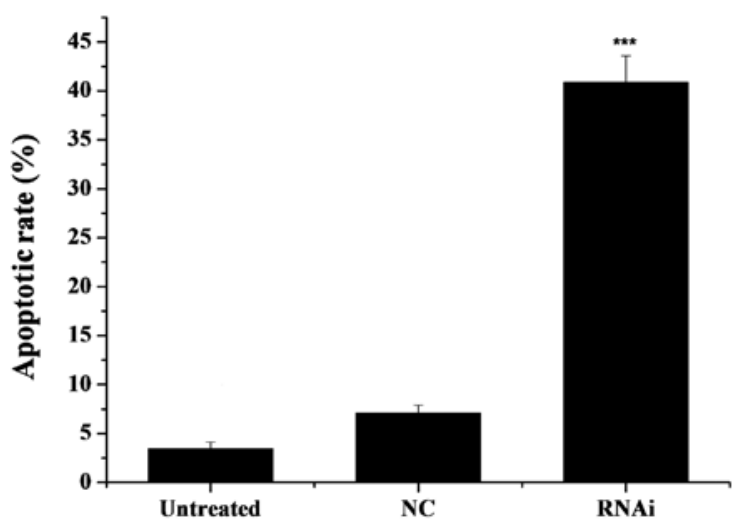

Figure 6. Apoptosis assay of U87 malignant glioma (MG) cells using the Annexin V-Cy3 detectionkit.Untreated, untransfected cells; NC, cellstransfected with the pGPU6-DIAPH1-NC plasmid as the mDRF1 knockdown-negative control group; RNAi, cells transfected with the pGPU6-DIAPH1-1 plasmid, as the mDRF1 knockdown group. Data are shown as means $\pm \mathrm{SE},{ }^{* * * *} \mathrm{P}<0.001$.

Knockdown of mDRF1 promotes cell apoptosis. After a 48-h transfection, we detected the apoptotic rate using the

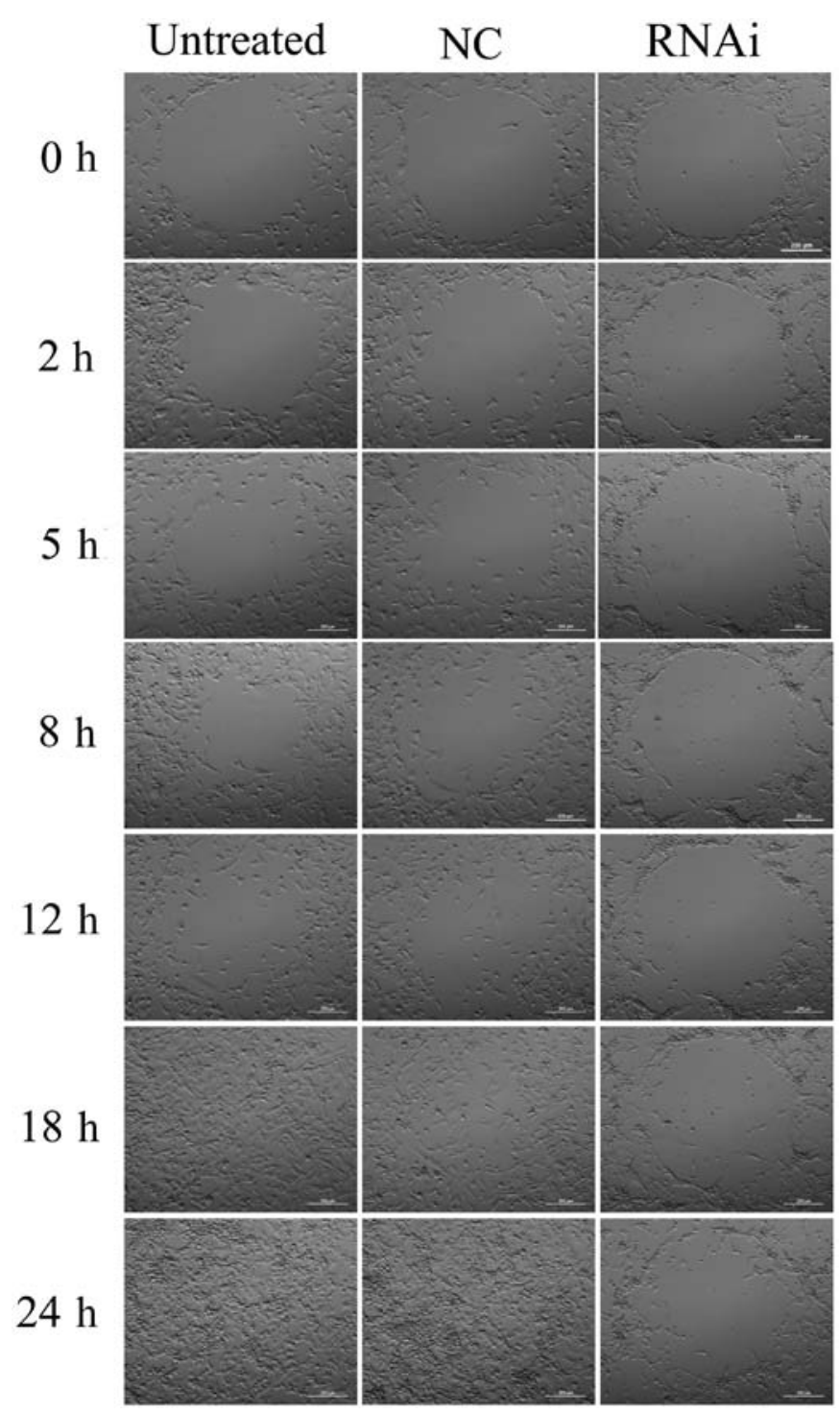

Figure 7. Wound healing assay. There are more cells migrating to the central open area in the untreated and the mDRF1 knockdown-negative control groups compared with the RNAi group $24 \mathrm{~h}$ after wound formation. Scale bar, $200 \mu \mathrm{m}$.

Annexin V-Cy3 apoptosis detection kit. As observed under a fluorescence microscope, the cells with red fluorescence were undergoing apoptosis. As illustrated in Fig. 6, the rate of apoptosis was $41.86 \%$ in the mDRF1 knockdown group, $3.87 \%$ in the untreated group and $7.54 \%$ in the mDRF1 knockdownnegative group. In contrast to the latter two negative control groups, the apoptotic rate was markedly higher in the U87 MG cells transfected with the pGPU6-DIAPH1-1 plasmid (mDRF1 knockdown goup). There were limited differences between the two negative control groups. These results reveal that the knockdown of mDRF1 promotes apoptosis in the U87 MG cell line.

Knockdown of mDRF1 inhibits cell migration. To examine the role of mDRF1 in U87 MG cell migration, we performed a wound healing assay. As shown in Fig. 7, cells in the mDRF1 knockdown group showed a decreased ability to migrate compared with the cells in the negative control group. Cells in the untreated and the mDRF1 knockdown-negative control 


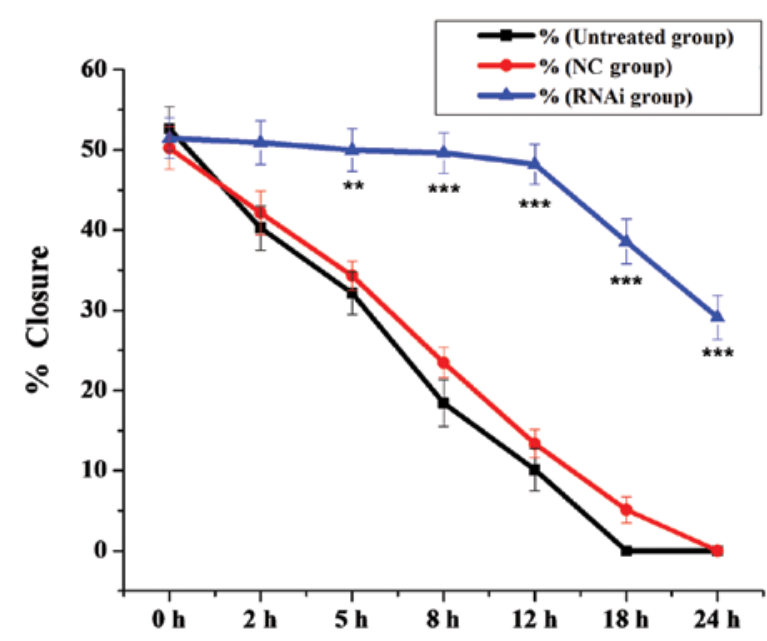

Figure 8 . Rates of cell migration into the open area, as estimated by the percentage of wound closure in the wound healing assay. A significant difference was observed between the RNAi (mDRF1 knockdown) group and the two negative control groups (NC, mDRF1 knockdown-negative; untreated, untransfected cells). Data are shown as means $\pm \mathrm{SE},{ }^{* *} \mathrm{P}<0.01,{ }^{* * *} \mathrm{P}<0.001$.

(NC) groups exhibited strong migration, so that the open areas were completely filled and reached saturation within $24 \mathrm{~h}$. By contrast, cells in the mDRF1 knockdown group showed a markedly slower migration rate, and even arrested motility. Twenty-four hours later, cells with the silenced mDRF1 copy were still unable to migrate to the open area in the center of the wells and a higher number of them died. The motility of the U87 MG cells decreased linearly with the decrease in the expression of mDRF1. Statistical comparisons of cell migration rates among the three groups, estimated from the ability of the cells to migrate into the central area, are shown in Fig. 8. The negative control groups showed a significantly faster migration rate than the mDRF1 knockdown group, which indicates that the silencing of mDRF1 reduces the motility of U87 MG cells.

Knockdown of $m D R F 1$ reduces the invasive ability of $U 87 M G$ cells. In the Transwell invasion assay, an ECM gel was used to mimic the ECM surrounding the glioma cells, and thereby, the in vitro tumor microenvironment. Following incubation of the U87 MG cells in the Transwell chamber for $24 \mathrm{~h}$, there was a greater number of cells that crossed the membranes in the control groups (untreated and mDRF1 knockdown-negative) compared with the mDRF1 knockdown/RNAi group (Fig. 9). The average number of invasive cells that crossed the membrane was approximately 125 in the untreated control group, approximately 105 in the NC (mDRF1 knockdownnegative) group and only 20 in the RNAi group, as shown in Fig. 10. Statistical analysis of these data revealed a significant difference between the two negative control groups and the RNAi group. No statistical difference was found between the two negative control groups. We conclude that the silencing of mDRF1 can reduce the invasive ability of the U87 MG cells and may be involved in the process of glioma cell invasion.

Knockdown of $m D R F 1$ reduces invadopodia formation. The results presented above indicated that MDRF1 may play an important role in the invasion and metastasis of human
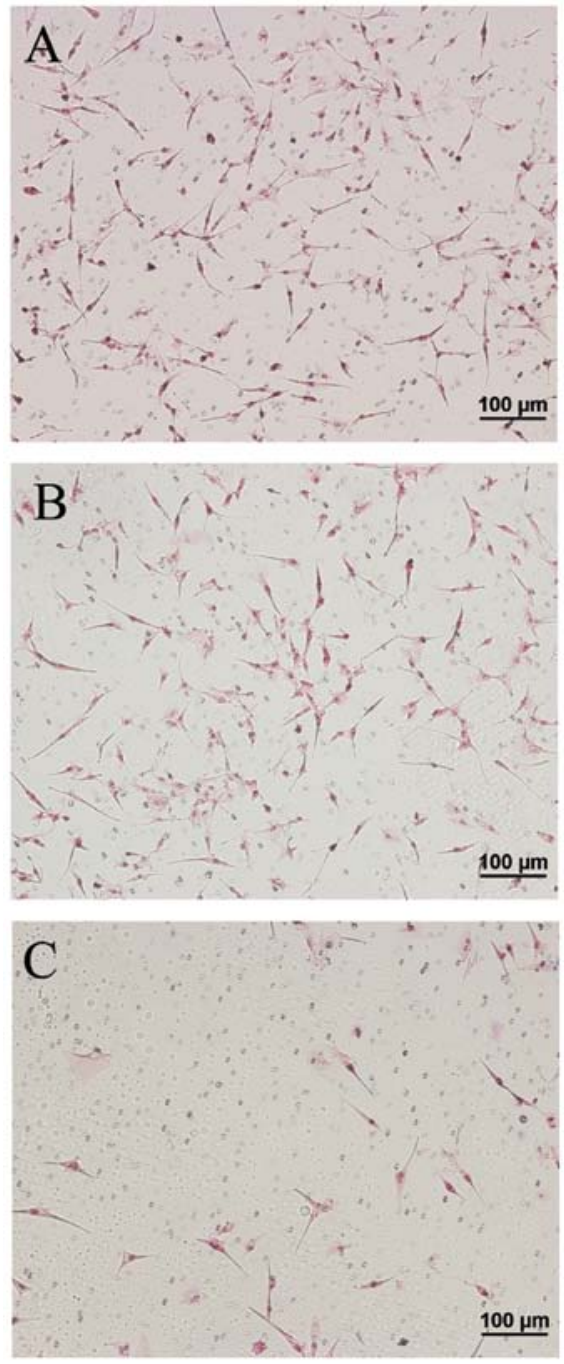

Figure 9. Transwell assay. (A-C) Cells from the three groups penetrating the membranes $24 \mathrm{~h}$ after seeding in the Transwell upper chamber. (A) Untransfected U87 malignant glioma (MG) cells (untreated group). (B) U87 MG cells transfected with the pGPU6-DIAPH1-NC plasmid (mDRF1 knockdown-negative group). (C) U87 MG cells transfected with the pGPU6DIAPH1-1 plasmid (mDRF1 knockdown/RNAi group). Scale bar, $100 \mu \mathrm{m}$.

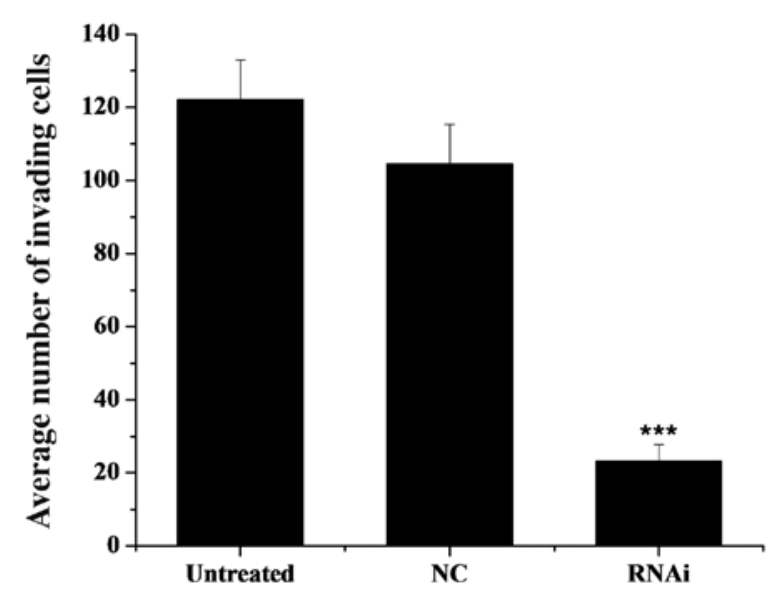

Figure 10. Average number of invading cells from the Transwell assay. There were 125 cells invading through the membrane in the untreated group (untransfected cells), as compared to 20 cells in the RNAi (mDRF1 knockdown) group. The difference between the RNAi and the two negative control groups (untreated, untransfected cells; NC, mDRF1 knockdown-negative group) was significant. Data are shown as means $\pm \mathrm{SE},{ }^{* * *} \mathrm{P}<0.001$. 
A

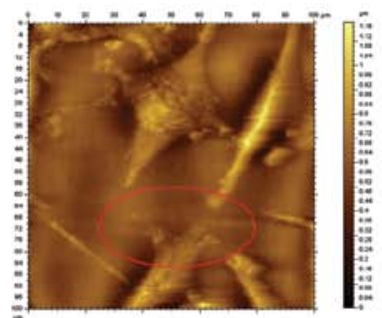

C

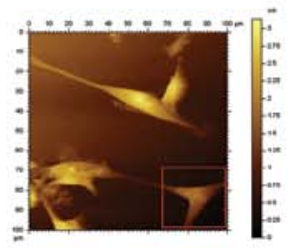

A-3D

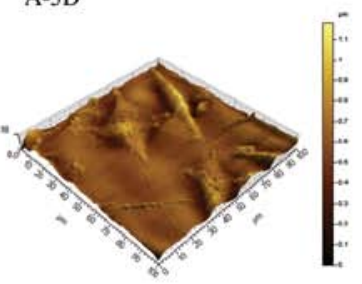

C-3D

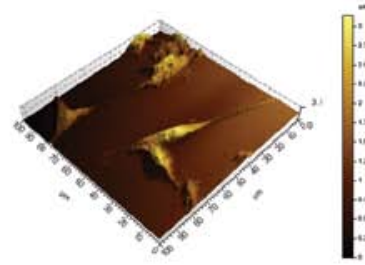

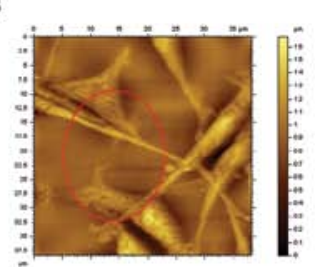

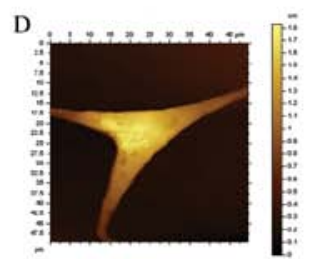

B-3D

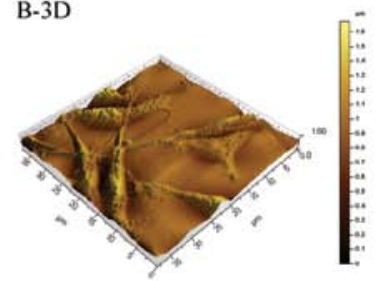

D-3D

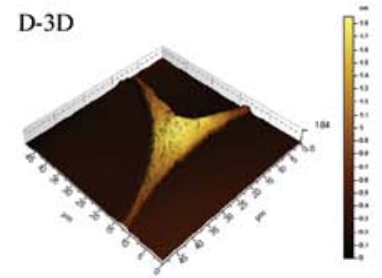

Figure 11. Invadopodia formation in U87 malignant glioma (MG) cells, observed under an atomic force microscope. (A and (A-3D) Invadopodia appear at the edge of the untreated U87 MG cells. (B and (B-3D) U87 cells transfected with the pGPU6-DIAPH1-NC plasmid also formed invadopodia. (C, C-3D, D and D-3D) U87 MG cells transfected with the pGPU6-DIAPH1-1 plasmid did not form invadopodia.

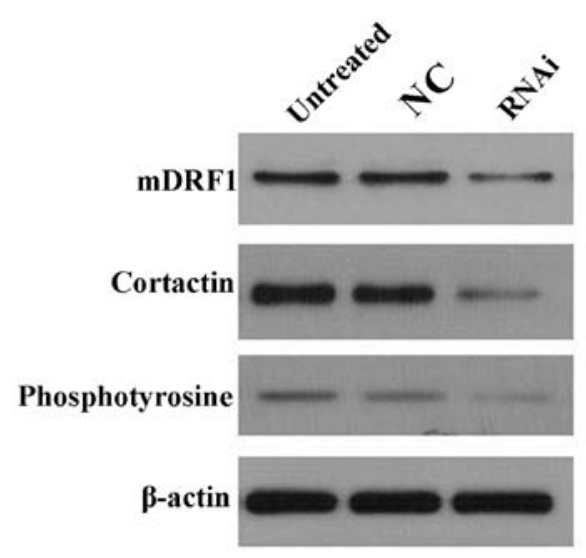

Figure 12. Protein levels of mammalian diaphanous-related formin 1 (mDRF1), cortactin and phosphotyrosine analyzed by western blot analysis. Following the silencing of mDRF1, the protein levels of mDRF1, cortactin and phosphotyrosine were reduced. The level of the $\beta$-actin gene was used for normalization.

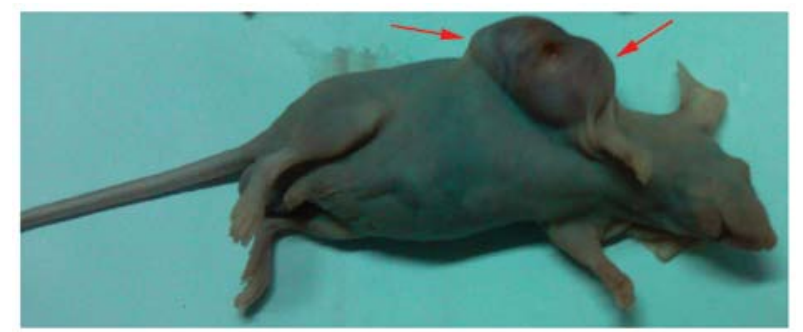

Figure 13. Representative U87 malignant glioma (MG) cell-derived tumor (red arrows) 40 days after implantation in a nude mouse.

U87 MG cells. In a variety of highly invasive tumor cells, invadopodia are the main structure degrading the ECM during invasion and migration. We therefore explored the effects of the silencing of mDRF1 on the formation of invadopodia in the U87 MG cell line. We used the contact mode to scan the glioma cells by atomic force microscopy (gas phase).

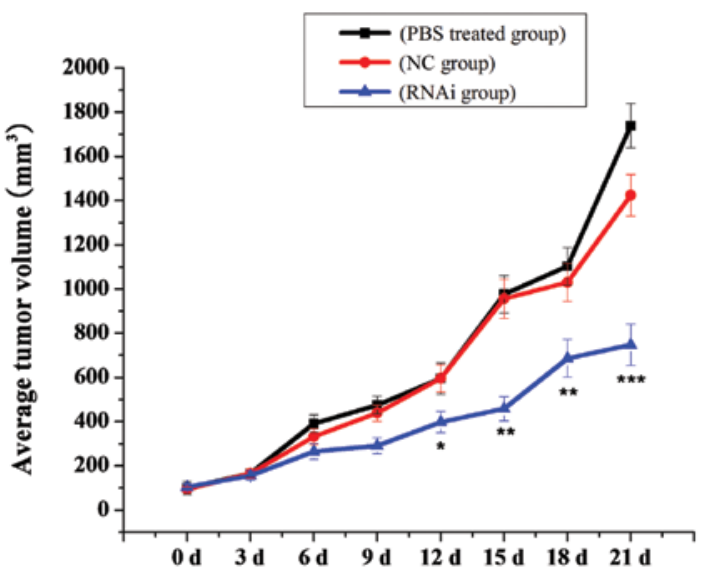

Figure 14. Average transplanted tumor size. After the formation of tumors, we injected PBS, pGPU6-DIAPH1-NC or pGPU6-DIAPH1-1 and observed tumor growth every two days. There was a significant difference between the RNAi (mDRF1 knockdown) group and the two negative control groups (PBStreated group; NC, mDRF1 knockdown-negative group). Data are shown as the means $\pm \mathrm{SE},{ }^{*} \mathrm{P}<0.05,{ }^{* *} \mathrm{P}<0.01,{ }^{* * *} \mathrm{P}<0.001$.

In the U87 MG cells that were not transfected, a large number of invasive branches, showing a slender filamentous structure, was observed at the cell edge, while cell morphology was normal (Fig. 11A and A-3D). Figs. 11A-, B-, C- and D-3D display the relationship between the cell and the basement (bottom of the cell culture dish). One can observe that the cells inlay the basement in an 'imbedded' phenotype in the untreated group (Fig. 11A-3D). Similarly, a high number of invadopodia appeared at the cell edge in an imbedded phenotype in the mDRF1 knockdown-negative group (Fig. 11B and B-3D). By contrast, in the mDRF1 knockdown group, shown in Fig. 11C and D, a smooth cell edge with no invadopodia was observed. In addition, the cell shape appeared changed and at times thinner. Cells at the surface of the basement displayed a 'suspended' phenotype (Fig. 11C-3D and D-3D ). These results demonstrate that mDRF1 is involved in the formation of invadopodia in U87 MG cells. 


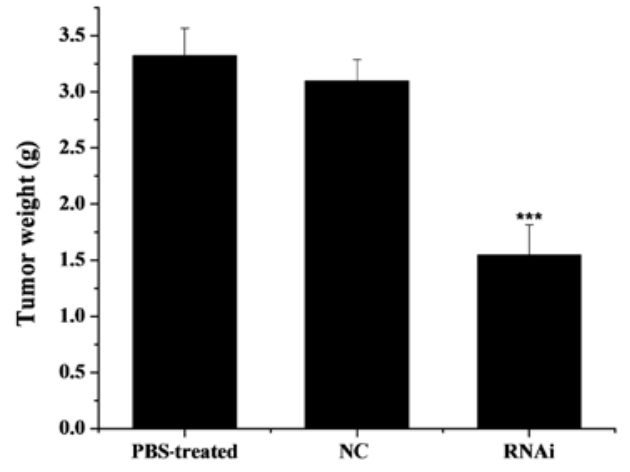

Figure 15. Average weight of the transplanted tumor. After observation for 21 days, the nude mice were sacrificed and the weight of the transplanted tumor tissue was recorded. There was a significant difference between the RNAi (mDRF1 knockdown) group and the two negative control groups (PBS-treated group; NC, mDRF1 knockdown-negative group).control groups. Data are shown as the means $\pm \mathrm{SE},{ }^{* * * *} \mathrm{P}<0.001$.

mDRF1 knockdown leads to reduced expression of cortactin and phosphotyrosine. The results from atomic force microscopy showed that the reduction in mDRF1 expression was accompanied by an absence of invadopodia. A previous study showed that cortactin and phosphotyrosine are abundant in the invadopodia (16). Therefore, changes in cortactin and phosphotyrosine expression levels were examined to explore the association between $\mathrm{mDRF} 1$ and these two proteins. Western blot analysis (Fig. 12) revealed that when the expression of mDRF1 decreased, and that the expression of cortactin and phosphotyrosine was reduced as well. Thus, we concluded that the silencing of the mDRF1 protein can affect the expression of cortactin and phosphotyrosine.

Knockdown of $m D R F 1$ expression suppresses the growth of U87 MG-derived tumors in nude mice. In order to further explore the function of mDRF1, we established the transplanted tumor model using nude mice transplanted with human U87 MG cells (Fig. 13). As shown in Fig. 14, there was a significant inhibitory effect on tumor growth in the mDRF1 knockdown group. By contrast, rapid tumor growth was observed in the negative control groups. Twenty-one days following these observations, the weight of the transplanted tumor was lower in the mDRF1 knockdown group compared with the control groups (Fig. 15). These data suggest that the silencing of mDRF1 inhibits tumor growth in nude mice.

\section{Discussion}

mDRF1 is a multi-domain protein. A point mutation in the gene (also termed DIAPH1) has been linked to non-syndromic sensorineural hearing loss (17). In addition, the abnormally high expression of mDRF1 in a variety of cancer types plays an important role in the formation of stress fibers, filopodia and microtubules, as well as in focal adhesion. To our knowledge, there is no study to date that has evaluated the roles of mDRF1 in glioma.

We previously conducted a microarray analysis of tumor-related genes and demonstrated that the expression of $m D R F 1 / D I A P H 1$ in glioma is up to 6.583-fold higher compared with healthy human brain tissue (18). Lizárraga et al (10) reported that upon the downregulation of mDRF1 in malignant breast cancer cells (MDA-MB-231), invadopodia formation was significantly reduced. Moreover, the study by Carramusa et al (19) indicated that mDRF1 can affect crosslinking between cells by regulating the synthesis of E-cadherin. In malignant breast cancer cells, the silencing of mDRF1 inhibited the E-cadherin protein located at the cell-cell junctions and thereby significantly reduced the adhesion between adjacent cells. Transfecting the cells with a plasmid, inducing the high expression of mDRF1, restored cross-linking between adjacent cells. These studies provide strong evidence that mDRF1 represents a promising target molecule in the treatment of cancer and suggest that it is involved in the invasion and metastasis of tumor cells.

In the present study, we successfully engineered U87 MG cells with a silenced copy of the mDRF1 gene by transfection with the pGPU6-DIAPH1-1 plasmid. Western blot analysis confirmed that the protein level of mDRF1 was reduced in these cells. Kato et al (20) reported that the reduced expression of mDRF1 inhibited spindle assembly and the formation of midbodies in HeLa cells. Moreover, altering the expression of mDRF1 can disturb the cell cycle and cell proliferation. The survival rate of the mDRF1 knockdown group in our study was lower compared to that of the control groups, indicating that the untreated cells had a stronger proliferative ability compared with the mDRF1-silenced ones. Upon the silencing of $\mathrm{mDRF} 1$, the number of apoptotic cells increased. The results from both assays are consistent with those from the study by Kato et al (20).

An earlier study demonstrated that mDRF1 expression correlates with cell invasion, with invasive ability increasing linearly with the increase in mDRF1 expression (10). Our results are in agreement with this report. Following the silencing of mDRF1 in the U87 MG cells, the cell invasive ability was reduced compared with the healthy cells. The results from the wound healing assay, where the migration of the mDRF1 knockdown cell group appeared reduced, further support this conclusion.

The molecular mechanisms underlying the process of invasion and migration in glioma cells are complex, involving numerous intracellular signaling pathways. From these, changes in cell morphology and invadopodia formation are believed to be regulated by the Rho GTP pathway and particularly by the proteins, RhoA, Rac1 and Cdc42 (21,22). This pathway plays an important role at the onset of tumor cell invasion and metastasis. Regulation, by mDRF1, of the invadopodia formation process has been reported in human breast cancer cells (10). Similarly, in our study, the U87 MG cells in which the mDRF1 protein was silenced had a reduced number of invadopodia. In the absence of mDRF1, the invasive and metastatic ability of the tumor cells appeared reduced, a result which warrants effective blocking of tumor cells from spreading to surrounding areas. However, the signaling pathway(s) through which mDRF1 exerts its effects remain to be fully elucidated.

The invasion of surrounding tissue by malignant tumor cells is the most important step initiating the process of cancer metastasis, further facilitated by the degradation of ECM barriers $(23,24)$. However, since the molecular mechanisms underlying tumor cell invasion and metastasis are complex, 
mDRF1 may regulate the motility of tumor cells in combination with other components, such as MMP-family proteins and E-cadherins (25).

In conclusion, our study demonstrates that mDRF1 is highly expressed in human U87 MG cells. Upon the knockdown of mDRF1 in these cells, the survival rate was reduced compared with the controls. The apoptotic rate of the cells in which mDRF1 was knocked down was higher than that of the control cells. The motility and invasive ability of the control cells was higher compared with that of the cells in which mDRF1 was knocked down. The formation of invadopodia was reduced in the mDRF1-silenced cells. In conclusion, mDRF1 plays a decisive role in the processes of proliferation, apoptosis, migration, invasive ability and invadopodia formation in U87 MG cells. These results indicate that the mDRF1 protein may be a good target for the treatment of glioma. Our study provides a novel prospect for the development of improved treatment strategies for glioma, through the use of the mDRF1 protein.

\section{Acknowledgements}

This study was supported by the Innovation Program of Shanghai Municipal Education Commission (grant no. 12ZZ100), the National Basic Research Program of China (grant no. 2010CB529806), the Leading Academic Discipline Project of Shanghai Municipal Education Commission 'Molecular Physiology'.

\section{References}

1. Louis DN, Ohgaki H, Wiestler OD, et al: The 2007 WHO classification of tumours of the central nervous system. Acta Neuropathol 114: 97-109, 2007.

2. Lee Y, Scheck AC, Cloughesy TF, et al: Gene expression analysis of glioblastomas identifies the major molecular basis for the prognostic benefit of younger age. BMC Med Genomics 1: 52 , 2008.

3. Miller G: Brain cancer. a viral link to glioblastoma? Science 323: 30-31, 2009.

4. Mentlein R, Hattermann $\mathrm{K}$ and Held-Feindt J: Lost in disruption: role of proteases in glioma invasion and progression. Biochim Biophys Acta 1825: 178-185, 2012.

5. Stupp R, Hegi ME, Mason WP, van den Bent MJ, et al: Effects of radiotherapy with concomitant and adjuvant temozolomide versus radiotherapy alone on survival in glioblastoma in a randomised phase III study: 5-year analysis of the EORTC-NCIC trial. Lancet Oncol 10: 459-466, 2009.

6. Goode BL and Eck MJ: Mechanism and function of formins in the control of actin assembly. Annu Rev Biochem 76: 593-627, 2007.
7. Castrillon DH and Wasserman SA: Diaphanous is required for cytokinesis in Drosophila and shares domains of similarity with the products of the limb deformity gene. Development 120 : 3367-3377, 1994

8. Zhu XL, Liang L and Ding YQ: Overexpression of FMNL2 is closely related to metastasis of colorectal cancer. Int J Colorectal Dis 23: 1041-1047, 2008.

9. DeWard AD, Eisenmann KM, Matheson SF and Alberts AS: The role of formins in human disease. Biochim Biophys Acta 1803: 226-233, 2010.

10. Lizárraga F, Poincloux R, Romao M, et al: Diaphanous-related formins are required for invadopodia formation and invasion of breast tumor cells. Cancer Res 69: 2792-2800, 2009.

11. Buccione R, Caldieri G and Ayala I: Invadopodia: specialized tumor cell structures for the focal degradation of the extracellular matrix. Cancer Metastasis Rev 28: 137-149, 2009.

12. Yamaguchi H, Pixley F and Condeelis J: Invadopodia and podosomes in tumor invasion. Eur J Cell Biol 85: 213-218, 2006.

13. Stylli SS, Kaye AH and Lock P: Invadopodia: at the cutting edge of tumour invasion. J Clin Neurosci 15: 725, 2008.

14. Carreira S, Goodall J, Denat L, et al: Mitf regulation of Dia1 controls melanoma proliferation and invasiveness. Genes Dev 20: 3426-3439, 2006.

15. Schoumacher M, Louvard D and Vignjevic D: Cytoskeleton networks in basement membrane transmigration. Eur J Cell Biol 90: 93-99, 2011.

16. Bowden ET, Onikoyi E, Slack R, et al: Co-localization of cortactin and phosphotyrosine identifies active invadopodia in human breast cancer cells. Exp Cell Res 312: 1240-1253, 2006.

17. Drummond MC, Belyantseva IA, Friderici KH and Friedman TB: Actin in hair cells and hearing loss. Hear Res 288: 89-99, 2012.

18. Yang Y, Qiu Y, Ren W, Gong J and Chen F: An identification of stem cell-resembling gene expression profiles in high-grade astrocytomas. Mol Carcinog 47: 893-903, 2008.

19. Carramusa L, Ballestrem C, Zilberman Y and Bershadsky AD: Mammalian diaphanous-related formin Dial controls the organization of E-cadherin-mediated cell-cell junctions. J Cell Sci 120: 3870-3882, 2007.

20. Kato T, Watanabe N, Morishima Y, Fujita A, Ishizaki T and Narumiya S: Localization of a mammalian homolog of diaphanous, mDial, to the mitotic spindle in HeLa cells. J Cell Sci 114: 775-784, 2001

21. Tominaga T, Sahai E, Chardin P, McCormick F, Courtneidge SA and Alberts AS: Diaphanous-related formins bridge Rho GTPase and Src tyrosine kinase signaling. Mol Cell 5: 13-25, 2000.

22. Narumiya $S$, Tanji $M$ and Ishizaki T: Rho signaling, ROCK and mDia1, in transformation, metastasis and invasion. Cancer Metastasis Rev 28: 65-76, 2009.

23. Weaver AM: Invadopodia: specialized cell structures for cancer invasion. Clin Exp Metastasis 23: 97-105, 2006.

24. Yamaguchi H: Pathological roles of invadopodia in cancer invasion and metastasis. Eur J Cell Biol 91: 902-907, 2012.

25. Kobielak A, Pasolli HA and Fuchs E: Mammalian formin-1 participates in adherens junctions and polymerization of linear actin cables. Nat Cell Biol 6: 21-30, 2004. 